\title{
INTEGRABILITY CONDITIONS FOR THE BIANCHI IDENTITIES AS TRANSFORMATIONS IN SCHWARZSCHILD SPACE-TIME
}

\author{
J. F. Q. FERNANDES ${ }^{1}$ and A. W.-C. LUN ${ }^{1}$
}

(Received 27 February 1995)

\begin{abstract}
We investigate the relationship between the Bardeen-Press and the Regge-Wheeler equations for perturbations of the Schwarzschild geometry. We examine how tetrad and coordinate gauge invariant Regge-Wheeler field quantities arise naturally from the perturbed Bianchi identities in the modified Newman-Penrose (compacted spincoefficient) formalism. The integrability conditions for the Bianchi identities then provide the transformation identities relating these quantities to the Bardeen-Press quantities. The relationships between the Bardeen-Press quantities of opposite spin-weight also arise naturally in our approach.
\end{abstract}

\section{Introduction}

In his analysis of the nonspherical perturbations of the Schwarzschild black hole, Price $[8,9]$ found that the imaginary part of the perturbed Newman-Penrose (NP) Weyl scalar $\psi_{2 B}$ satisfies the Regge-Wheeler [10] (RW) equation. (In this article we denote perturbation quantities by a subscript $B$.) Subsequently, Lun and Fackerell [6] and Lun [5] were able to show that, upon the adoption of a suitable gauge, $\psi_{2 B}$ itself can be made to satisfy the RW equation.

More recently, Chandrasekhar [2] has investigated the perturbation equations extensively, and show how the Bardeen-Press [1] (BP) equations can be transformed to the RW equation (see also Sasaki and Nakamura [11]). His analysis is based on the transformations of differential operators. It is our aim to show that these results may be derived from the perturbed Bianchi identities.

We use the modified Newman-Penrose (compacted spin-coefficient) formalism [7]. Since the perturbed Bianchi identities are gauge invariant, $f_{u}\left(R_{j[k l ; m]}^{i}\right)_{B}=0$ (see

${ }^{1}$ Department of Mathematics, Monash University, Clayton, Victoria 3168, Australia

(C) Australian Mathematical Society 1999, Serial-fee code 0334-2700/99 
Lun [5]), the field quantities which arise naturally from our analysis are also gauge invariant, that is, invariant under infinitesimal coordinate and infinitesimal Lorentz transformations. We will show how the Bianchi identities give rise not only to the BP equations, but also to two gauge independent RW equations. Consequently we present gauge (and tetrad) invariant RW field quantities, which are related to $\psi_{2 B}$. We will also show how the integrability conditions for the Bianchi identities provide, in a natural way, the transformations between the BP and RW equations. The relationships between the BP quantities of opposite spin-weight, commonly referred to as the "Teukolsky-Starobinsky" identities, also arise naturally in our approach. Some of the results presented here have also appeared elsewhere [4]. Here we extend the results in Fernandes and Lun [4] and also provide some of the missing detail.

\section{Gravitational perturbations}

The Schwarzschild space-time may be described in the compacted spin-coefficient formalism using the null tetrad:

$$
\begin{array}{r}
l^{j}=\frac{1}{\Delta}\left(r^{2}, \Delta, 0,0\right), \\
n^{j}=\frac{1}{2 r^{2}}\left(r^{2},-\Delta, 0,0\right), \\
m^{j}=\frac{1}{r \sqrt{2}}(0,0,1, i \operatorname{cosec} \vartheta),
\end{array}
$$

where $\Delta=r^{2}-2 M r$. Then

$$
\begin{gathered}
\kappa=\sigma=\lambda=\nu=\varepsilon=\pi=\tau=\Phi_{a b}=\psi_{0}=\psi_{1}=\psi_{3}=\psi_{4}=0, \\
\varrho=\bar{\varrho}=-\frac{1}{r}, \mu=\bar{\mu}=-\frac{\Delta}{2 r^{3}}, \alpha=-\beta=-\frac{\cot \vartheta}{2 r \sqrt{2}}, \gamma=\frac{M}{2 r^{2}}, \psi_{2}=-\frac{M}{r^{3}}, \\
\mathrm{p} \varrho=\varrho^{2}, \mathrm{p}^{\prime} \varrho=-\varrho \mu-\psi_{2}, \mathrm{p} \mu=\varrho \mu+\psi_{2}, \mathrm{p}^{\prime} \mu=-\mu^{2}, \\
\partial \psi_{2}=\partial \mu=\partial \varrho=0, \\
\mathrm{p} \psi_{2}=3 \varrho \psi_{2}, \quad \mathrm{p}^{\prime} \psi_{2}=-3 \mu \psi_{2} .
\end{gathered}
$$

For clarity, some of the technical detail of the present section will be deferred to the appendices.

The fundamental equations for the gravitational perturbations are the (gauge invariant) perturbed Bianchi identities ${ }^{2}$ which become, after linearization:

$$
(\mathrm{p}-4 \varrho) \psi_{1 B}=\sigma^{\prime} \psi_{0 B}-3 \kappa_{B} \psi_{2},
$$

\footnotetext{
${ }^{2}$ We refer the reader to Penrose and Rindler [7] or Chandrasekhar [2] for a full description of the Bianchi and Ricci identities.
} 


$$
\begin{gathered}
(\mathrm{p}-3 \varrho) \psi_{2 B}+\left(\mathrm{p}_{B}-3 \varrho_{B}\right) \psi_{2}=\partial^{\prime} \psi_{1 B}, \\
(\mathrm{p}-2 \varrho) \psi_{3 B}=\partial^{\prime} \psi_{2 B}+\left({\sigma_{B}^{\prime}}^{\prime}+3 \pi_{B}\right) \psi_{2}, \\
(\mathrm{p}-\varrho) \psi_{4 B}=\partial^{\prime} \psi_{3 B}-3 \lambda_{B} \psi_{2}, \\
\left(\mathrm{p}^{\prime}+\mu\right) \psi_{0 B}=\partial \psi_{1 B}+3 \sigma_{B} \psi_{2}, \\
\left(\mathrm{p}^{\prime}+2 \mu\right) \psi_{1 B}=\partial \psi_{2 B}+\left(\bar{\partial}_{B}-3 \tau_{B}\right) \psi_{2}, \\
\left(\mathrm{p}^{\prime}+3 \mu\right) \psi_{2 B}+\left(\mathrm{p}_{B}^{\prime}+3 \mu_{B}\right) \psi_{2}=\partial \psi_{3 B}, \\
\left(\mathrm{p}^{\prime}+4 \mu\right) \psi_{3 B}=\partial \psi_{4 B}+3 \nu_{B} \psi_{2} .
\end{gathered}
$$

Furthermore, the following (Ricci) identities relating to the spin coefficients will be useful:

$$
\begin{gathered}
\mathrm{p} \varrho_{B}+\mathrm{p}_{B} \varrho-\partial^{\prime} \kappa_{B}=2 \varrho \varrho_{B}, \\
\mathrm{p} \sigma_{B}-\partial \kappa_{B}=2 \sigma_{B} \varrho+\psi_{0 B}, \\
\mathrm{p} \lambda_{B}-\partial^{\prime} \pi_{B}=\varrho \lambda_{B}+\mu \bar{\sigma}_{B}, \\
\mathrm{p} \mu_{B}+\mathrm{p}_{B} \mu-\partial \pi_{B}=\mu \bar{\varrho}_{B}+\mu_{B} \varrho+\psi_{2 B}, \\
\mathrm{p}^{\prime} \lambda_{B}-\bar{\partial}^{\prime} \nu_{B}=-2 \lambda_{B} \mu-\psi_{4 B}, \\
\partial_{B} \varrho+\partial_{B}-\partial^{\prime} \sigma_{B}=-\psi_{1 B}, \\
\partial \lambda_{B}-\bar{\partial}_{B}^{\prime} \mu-\partial^{\prime} \mu_{B}=-\psi_{3 B}, \\
\partial \nu_{B}-\mathrm{p}^{\prime} \mu_{B}-\mathrm{p}_{B}^{\prime} \mu=2 \mu \mu_{B}, \\
\partial \tau_{B}-\mathrm{p}^{\prime} \sigma_{B}=\mu \sigma_{B}+\varrho \bar{\lambda}_{B}, \\
\mathrm{p}^{\prime} \varrho_{B}+\mathrm{p}_{B}^{\prime} \varrho-\bar{\partial}^{\prime} \tau_{B}=-\varrho \bar{\mu}_{B}-\varrho_{B} \bar{\mu}-\psi_{2 B} .
\end{gathered}
$$

From the integrability conditions on the perturbed Bianchi identities we obtain four wave equations as follows. Operating with $(p-3 Q)$ on (9) and with $\partial$ on (5), and using the appropriate NP commutation relation in conjunction with (2), we find

$$
\begin{aligned}
& {\left[(\mathrm{p}-3 \varrho)\left(\mathrm{p}^{\prime}+3 \mu\right)-\partial \boldsymbol{\partial}^{\prime}\right] \psi_{2 B}} \\
& \quad+\left[(\mathrm{p}-3 \varrho)\left(\mathrm{p}_{B}^{\prime}+3 \mu_{B}\right)-\partial\left(\bar{\partial}_{B}^{\prime}+3 \pi_{B}\right)\right] \psi_{2}=0 .
\end{aligned}
$$

Now (14) and (2) allow the above to be cast into the more convenient form (with no explicit derivatives of perturbed spin coefficients)

$$
\begin{aligned}
& {\left[(\mathrm{p}-3 \varrho)\left(\mathrm{p}^{\prime}+3 \mu\right)-\not \boldsymbol{\sigma}^{\prime}+3 \psi_{2}\right] \psi_{2 B}} \\
& +\left[(\mathrm{p}-3 \varrho) \mathrm{p}_{B}^{\prime}-\partial \bar{\partial}_{B}^{\prime}-3 \mathrm{p}_{B} \mu+3 \varrho \mu_{B}+3 \mu \bar{\varrho}_{B}\right] \psi_{2}=0 \text {. }
\end{aligned}
$$

Likewise, we may derive from (4), (8) and (20)

$$
\left[\left(\mathrm{p}^{\prime}+3 \mu\right)(\mathrm{p}-3 \varrho)-\bar{\partial}^{\prime} \partial+3 \psi_{2}\right] \psi_{2 B}
$$




$$
+\left[\left(\mathrm{p}^{\prime}+3 \mu\right) \mathrm{p}_{B}-\sigma^{\prime} \partial_{B}+3 \mathrm{p}_{B}^{\prime} \varrho+3 \varrho_{B} \mu+3 \varrho \bar{\mu}_{B}\right] \psi_{2}=0
$$

and from (6), (10), (15) and (3), (7), (12), respectively: (Chandrasekhar [2])

$$
\begin{aligned}
& {\left[\left(\mathrm{p}^{\prime}+5 \mu\right)(\mathrm{p}-\varrho)-\partial^{\prime} \partial-3 \psi_{2}\right] \psi_{4 B}=0} \\
& {\left[(\mathrm{p}-5 \varrho)\left(\mathrm{p}^{\prime}+\mu\right)-\partial \bar{\partial}^{\prime}-3 \psi_{2}\right] \psi_{0 B}=0 .}
\end{aligned}
$$

With the tetrad given in (1), (24) and (25) are the usual spin -2 and spin $+2 \mathrm{BP}$ equations, respectively.

Using the technique outlined above, the perturbed Bianchi identities also give rise to the following four (transformation) identities: (refer to the Appendix A1 for a worked example)

$$
\begin{aligned}
& (\mathrm{p}-3 \varrho)(\mathrm{p}-\varrho) \psi_{4 B}=\bar{\sigma}^{\prime} \mathrm{\partial}^{\prime} \psi_{2 B}+\left(\mathrm{\sigma}^{\prime} \mathrm{\sigma}_{B}^{\prime}-3 \varrho \lambda_{B}-3 \mu \bar{\sigma}_{B}\right) \psi_{2}, \\
& \left(\mathrm{p}^{\prime}+5 \mu\right)\left(\mathrm{p}^{\prime}+3 \mu\right) \psi_{2 B}+\left[\left(\mathrm{p}^{\prime}+5 \mu\right) \mathrm{p}_{B}^{\prime}-3 \mathrm{p}_{B}^{\prime} \mu\right] \psi_{2}=\text { бठ } \psi_{4 B} \text {, } \\
& \left(\mathrm{p}^{\prime}+3 \mu\right)\left(\mathrm{p}^{\prime}+\mu\right) \psi_{0 B}=\partial \partial \psi_{2 B}+\left(\partial \bar{\partial}_{B}-3 \mu \sigma_{B}-3 \varrho \bar{\lambda}_{B}\right) \psi_{2} \text {, } \\
& (\mathrm{p}-5 \varrho)(\mathrm{p}-3 \varrho) \psi_{2 B}+\left[(\mathrm{p}-5 Q) \mathrm{p}_{B}+3 \mathrm{p}_{B} \varrho\right] \psi_{2}=\mathbf{g}^{\prime} \mathrm{J}^{\prime} \psi_{0 B} \text {. }
\end{aligned}
$$

The significance of these relations is two-fold. Firstly, they are gauge invariant (that is, invariant under infinitesimal coordinate and infinitesimal tetrad transformations). Secondly, they provide the required transformations between the BP and RW equations. To see this, we define the following quantities:

$$
\begin{aligned}
& \xi_{0}:=\frac{4 r^{4}}{(l-1) l(l+1)(l+2)} \partial \delta\left(\sigma^{\prime} \partial_{B}^{\prime}-3 \varrho \lambda_{B}-3 \mu \bar{\sigma}_{B}\right) \psi_{2}, \\
& \xi_{0}^{\prime}:=\frac{4 r^{4}}{(l-1) l(l+1)(l+2)} \tilde{\sigma}^{\prime} \boldsymbol{\partial}^{\prime}\left(\delta \tilde{\sigma}_{B}-3 \mu \sigma_{B}-3 \varrho \bar{\lambda}_{B}\right) \psi_{2}=\bar{\xi}_{0}, \\
& \xi_{-2}:=\frac{12 r^{4} \psi_{2}}{(l-1) l(l+1)(l+2)}\left(\varrho \mathrm{p}^{\prime}+\mu \mathrm{p}-2 \psi_{2}\right) \psi_{4 B}, \\
& \xi_{+2}:=\frac{12 r^{4} \psi_{2}}{(l-1) l(l+1)(l+2)}\left(-\mu \mathrm{p}-\varrho \mathrm{p}^{\prime}-2 \psi_{2}\right) \psi_{0 B}=\xi_{-2}^{\prime},
\end{aligned}
$$

( $l \geq 2$ for gravitational radiation). It is apparent that $\xi_{0}, \xi_{0}^{\prime}, \xi_{-2}$ and $\xi_{+2}$ are weighted quantities (see Penrose and Rindler [7]) of $(p, q)$ type $(0,0),(0,0),(-4,0)$ and $(4,0)$, respectively.

With these definitions it can be proved (refer to Appendix A1) that our transformation identities (26)-(29) become

$$
\begin{gathered}
(\mathrm{p}-3 \varrho)(\mathrm{p}-\varrho) \psi_{4 B}=\sigma^{\prime} \sigma^{\prime}\left(\psi_{2 B}+\xi_{0}\right), \\
\left(\mathrm{p}^{\prime}+5 \mu\right)\left(\mathrm{p}^{\prime}+3 \mu\right)\left(\psi_{2 B}+\xi_{0}\right)=\delta \partial\left(\psi_{4 B}+\xi_{-2}\right),
\end{gathered}
$$




$$
\begin{gathered}
\left(\mathrm{p}^{\prime}+3 \mu\right)\left(\mathrm{p}^{\prime}+\mu\right) \psi_{0 B}=\sigma \delta\left(\psi_{2 B}+\xi_{0}^{\prime}\right), \\
(\mathrm{p}-5 \varrho)(\mathrm{p}-3 \varrho)\left(\psi_{2 B}+\xi_{0}^{\prime}\right)=\sigma^{\prime}{\partial^{\prime}}^{\prime}\left(\psi_{0 B}+\xi_{+2}\right)
\end{gathered}
$$

and the wave equations (22) and (23) simplify greatly to give (see Appendix A2)

$$
\begin{aligned}
& {\left[(\mathrm{p}-3 \varrho)\left(\mathrm{p}^{\prime}+3 \mu\right)-\boldsymbol{\partial}^{\prime}+3 \psi_{2}\right]\left(\psi_{2 B}+\xi_{0}\right)=0,} \\
& {\left[\left(\mathrm{p}^{\prime}+3 \mu\right)(\mathrm{p}-3 \varrho)-\partial^{\prime} \partial+3 \psi_{2}\right]\left(\psi_{2 B}+\xi_{0}^{\prime}\right)=0 .}
\end{aligned}
$$

We are justified in referring to (22) and (23) as wave equations in the light of their amazing reduction to this form. In fact these equations are none other than the RW equation, after expansion in coordinates. An important feature of (38) and (39) (and also (34)-(37)) is that they are completely gauge invariant (refer to Appendix A3). The (RW) operators associated with (38) and (39) can be seen to be identical by making use of the commutators $\left[\mathrm{b}, \mathrm{p}^{\prime}\right]$ and $\left[\tilde{\partial}, \bar{\delta}^{\prime}\right]$, however the field quantities $\psi_{2 B}+\xi_{0}$ and $\psi_{2 B}+\xi_{0}^{\prime}$ are distinct, with $\xi_{0}$ connected to $\psi_{4 B}$ via (34) and $\xi_{0}^{\prime}=\bar{\xi}_{0}$ connected to $\psi_{0 B}$ via (36). We emphasize their difference here, since this will be significant for the identities relating BP quantities of opposite spin-weight later (see (54) and (55)). Hence the integrability conditions naturally provide two RW equations.

It can be proved that the quantities $\xi_{-2}$ and $\xi_{+2}$ satisfy the spin -2 and spin +2 BP equations:

$$
\begin{aligned}
& {\left[\left(\mathrm{p}^{\prime}+5 \mu\right)(\mathrm{p}-\varrho)-\partial^{\prime} \partial-3 \psi_{2}\right] \xi_{-2}=0} \\
& {\left[(\mathrm{p}-5 \varrho)\left(\mathrm{p}^{\prime}+\mu\right)-\partial^{\prime}-3 \psi_{2}\right] \xi_{+2}=0 .}
\end{aligned}
$$

Indeed one can prove the following commutation relation for quantities of type $(-4,0)$ :

$$
\begin{aligned}
& {\left[\left(\mathrm{p}^{\prime}+5 \mu\right)(\mathrm{p}-\varrho)-\mathrm{\partial}^{\prime} \mathrm{\partial}-3 \psi_{2}\right]\left[r^{4} \psi_{2}\left(\varrho \mathrm{p}^{\prime}+\mu \mathrm{p}-2 \psi_{2}\right)\right]} \\
& \quad=\left[r^{4} \psi_{2}\left(\varrho \mathrm{p}^{\prime}+\mu \mathrm{p}-2 \psi_{2}\right)\right]\left[\left(\mathrm{p}^{\prime}+5 \mu\right)(\mathrm{p}-\varrho)-\partial^{\prime} \partial-3 \psi_{2}\right] .
\end{aligned}
$$

This can be checked directly, making use of the appropriate NP commutators and the relations (2), but is self-evident when $\xi_{-2}$ is written in coordinate form:

$$
\xi_{-2}=\frac{12 M}{(l-1) l(l+1)(l+2)} \frac{\partial}{\partial t} \psi_{4 B} .
$$

So the operator

$$
r^{4} \psi_{2}\left(\varrho \mathbf{p}^{\prime}+\mu \mathrm{p}+\frac{1}{2}(p+q) \psi_{2}\right)
$$

corresponds to the $\partial_{t}$ Killing vector of the background space-time, for a quantity of type $(p, q)$. 
We have now achieved our primary aims. The $(0,0)$ quantities

$$
\begin{aligned}
& \widehat{\psi}_{2 B}=\psi_{2 B}+\xi_{0}, \quad \text { and } \\
& \widehat{\psi}_{2 B}^{\prime}=\psi_{2 B}+\xi_{0}^{\prime}
\end{aligned}
$$

are gauge invariant, and satisfy the RW equation. Equations (34)-(37) act as transformations between the RW and BP quantities. In particular, taking (34), we have after resolution into spin-weighted spherical harmonics,

$$
\widehat{\psi}_{2 B}=\frac{4 r^{4}}{(l-1) l(l+1)(l+2)} \partial \delta(\mathrm{p}-3 \varrho)(\mathrm{p}-\varrho) \psi_{4 B} .
$$

Applying the RW operator

$$
\left[(\mathrm{p}-3 \varrho)\left(\mathrm{p}^{\prime}+3 \mu\right)-\partial \bar{\sigma}^{\prime}+3 \psi_{2}\right]
$$

to this quantity, we derive (again by making repeated use of the NP commutation relationships and Equations (2)) a commutation relation for quantities of $(p, q)$ type $(-4,0)$ :

$$
\begin{aligned}
& {\left[(\mathrm{p}-3 \varrho)\left(\mathrm{p}^{\prime}+3 \mu\right)-\partial \boldsymbol{\sigma}^{\prime}+3 \psi_{2}\right]\left[r^{4} \boldsymbol{\partial}(\mathrm{p}-3 \varrho)(\mathrm{p}-\varrho)\right]} \\
& \quad=\left[r^{4} \partial \partial(\mathrm{p}-5 \varrho)(\mathrm{p}-3 \varrho)\right]\left[\left(\mathrm{p}^{\prime}+5 \mu\right)(\mathrm{p}-\varrho)-\boldsymbol{\partial}^{\prime} \boldsymbol{\partial}-3 \psi_{2}\right] .
\end{aligned}
$$

Hence assuming the spin $-2 \mathrm{BP}$ equation (24), we have the RW equation

$$
\left[(\mathrm{p}-3 \varrho)\left(\mathrm{p}^{\prime}+3 \mu\right)-\partial \boldsymbol{\partial}^{\prime}+3 \psi_{2}\right]\left(\widehat{\psi}_{2 B}\right)=0 \text {. }
$$

Conversely, from (35)

$$
\psi_{4 B}+\xi_{-2}=\frac{4 r^{4}}{(l-1) l(l+1)(l+2)} \sigma^{\prime} \boldsymbol{\sigma}^{\prime}\left(\mathrm{p}^{\prime}+5 \mu\right)\left(\mathrm{p}^{\prime}+3 \mu\right) \widehat{\psi}_{2 B}
$$

and we may derive the spin $-2 \mathrm{BP}$ equation for $\psi_{4 B}+\xi_{-2}$ from the RW equation, by operating on (48) with the BP operator

$$
\left[\left(\mathrm{p}^{\prime}+5 \mu\right)(\mathrm{p}-\varrho)-\boldsymbol{\sigma}^{\prime} \partial-3 \psi_{2}\right]
$$

and making use of the following commutation relation for quantities of type $(0,0)$ :

$$
\begin{aligned}
& {\left[\left(\mathrm{p}^{\prime}+5 \mu\right)(\mathrm{p}-\varrho)-\boldsymbol{\partial}^{\prime} \boldsymbol{\partial}-3 \psi_{2}\right]\left[r^{4} \boldsymbol{\partial}^{\prime} \boldsymbol{\partial}^{\prime}\left(\mathrm{p}^{\prime}+5 \mu\right)\left(\mathrm{p}^{\prime}+3 \mu\right)\right]} \\
& \quad=\left[r^{4} \boldsymbol{\partial}^{\prime} \boldsymbol{\partial}^{\prime}\left(\mathrm{p}^{\prime}+7 \mu\right)\left(\mathrm{p}^{\prime}+5 \mu\right)\right]\left[(\mathrm{p}-3 \varrho)\left(\mathrm{p}^{\prime}+3 \mu\right)-\delta \boldsymbol{\partial}^{\prime}+3 \psi_{2}\right]
\end{aligned}
$$

Similar results follow from (36) and (37), or alternatively, by applying the prime operator (Penrose and Rindler [7]) to the above. They give the relationships between the spin +2 BP equation (25) and the RW equation (39). 
Since $\psi_{2}$ is real, using (30) and (31), it can be seen that the two quantities

$$
\begin{aligned}
\widehat{\psi}_{2 B}-\widehat{\psi}_{2 B}^{\prime} & =2 i \operatorname{Im}\left(\xi_{0}\right), \quad \text { and } \\
\widehat{\psi}_{2 B}-\left(\widehat{\psi}_{2 B}^{\prime}\right) & =2 i \operatorname{Im}\left(\psi_{2 B}\right)
\end{aligned}
$$

are gauge invariant. These purely imaginary quantities also satisfy the RW equation. This is reminiscent of Price's [9] early result: Using the perturbed NP commutators repeatedly, and (16), (17), (4) and (9), we can show that the quantity $\operatorname{Im}\left(\xi_{0}\right)$ in $(50)$ reduces to the form

$$
\operatorname{Im}\left(\xi_{0}\right)=\frac{12 r^{4} \psi_{2}}{(l-1) l(l+1)(l+2)}\left(\varrho \mathbf{p}^{\prime}+\mu \mathrm{p}\right) \operatorname{Im}\left(\psi_{2 B}\right) .
$$

We have seen the operator $r^{4} \psi_{2}\left(\varrho \mathrm{p}^{\prime}+\mu \mathrm{p}\right)$ before - it corresponds to the $\partial_{t}$ Killing vector of the background space-time. It is interesting that the operator in (52) arises so naturally over and over in our results. Expanding (52) in coordinates reveals

$$
\operatorname{Im}\left(\xi_{0}\right)=\frac{12 M}{(l-1) l(l+1)(l+2)} \frac{\partial}{\partial t} \operatorname{Im}\left(\psi_{2 B}\right) .
$$

Now, the identities (34)-(37) also relate $\psi_{4 B}$ and $\psi_{0 B}$. We may derive, from our transformation identities, the following relationships in a straightforward way:

$$
\begin{aligned}
& (\mathrm{p}-7 \varrho)(\mathrm{p}-5 \varrho)(\mathrm{p}-3 \varrho)(\mathrm{p}-\varrho) \psi_{4 B} \\
& =\boldsymbol{\sigma}^{\prime} \boldsymbol{\partial}^{\prime} \boldsymbol{\partial}^{\prime} \boldsymbol{\partial}^{\prime}\left(\psi_{0 B}+\xi_{+2}\right)+(\mathrm{p}-7 \varrho)(\mathrm{p}-5 \varrho) \boldsymbol{\partial}^{\prime} \boldsymbol{\partial}^{\prime}\left(\xi_{0}-\xi_{0}^{\prime}\right) \text {, } \\
& \left(\mathrm{p}^{\prime}+7 \mu\right)\left(\mathrm{p}^{\prime}+5 \mu\right)\left(\mathrm{p}^{\prime}+3 \mu\right)\left(\mathrm{p}^{\prime}+\mu\right) \psi_{0 B}
\end{aligned}
$$

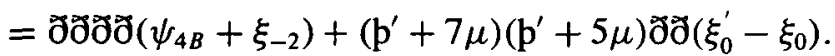

These identities allow, say, $\psi_{0 B}$ to be determined if $\psi_{4 B}$ is known. In this case, using (52), the identity (34), the complex conjugate of (36) and the higher order equation

$$
(\mathrm{p}-7 \varrho)(\mathrm{p}-5 \varrho)\left(\mathrm{p}^{\prime}+3 \mu\right)\left(\mathrm{p}^{\prime}+\mu\right) \psi_{0 B}=\partial^{\prime} \bar{\partial}^{\prime} \widetilde{\partial}^{\prime}\left(\psi_{0 B}+\xi_{+2}\right)
$$

(from (36) and (37)), we may eliminate reference to the term $\left(\xi_{0}-\xi_{0}^{\prime}\right)$ from (54), to get

$$
\begin{aligned}
& (\mathrm{p}-7 \varrho)(\mathrm{p}-5 \varrho)(\mathrm{p}-3 \varrho)(\mathrm{p}-\varrho)\left(\psi_{4 B}-\xi_{-2}\right) \\
& \quad=\boldsymbol{J}^{\prime} \boldsymbol{J}^{\prime} \boldsymbol{J}^{\prime} \boldsymbol{\partial}^{\prime}\left(\psi_{0 B}+\xi_{+2}\right)-3 \psi_{2}\left(\varrho \mathrm{p}^{\prime}+\mu \mathrm{p}+2 \psi_{2}\right)\left(\overline{\psi_{0 B}+\xi_{+2}}\right) .
\end{aligned}
$$

Now (57), together with its complex conjugate, imply, when expanded in coordinates and using the time dependence $e^{i \sigma t}$,

$$
\left[((l-1) l(l+1)(l+2))^{2}+144 M^{2} \sigma^{2}\right] \psi_{0 B}
$$




$$
=\mathscr{L}_{-1}^{\dagger} \mathscr{L}_{0}^{\dagger} \mathscr{L}_{1}^{\dagger} \mathscr{L}_{2}^{\dagger} \mathscr{D}_{0} \mathscr{D}_{0} \mathscr{D}_{0} \mathscr{D}_{0}\left(4 r^{4} \psi_{4 B}\right)+12 M i \sigma \mathscr{D}_{0} \mathscr{D}_{0} \mathscr{D}_{0} \mathscr{D}_{0}\left(4 r^{4} \overline{\psi_{4 B}}\right) \text {, }
$$

where the differential operators $\mathscr{L}_{n}, \mathscr{L}_{m}^{\dagger}, \mathscr{D}_{0}$ and $\mathscr{D}_{0}^{\dagger}$ are as defined in Chandrasekhar [2]. If certain assumptions are made about the complex conjugate of $\psi_{4 B}$, it is not difficult to show that (58) is in agreement with Chandrasekhar [2].

When written in coordinates, the identities (46) and (48) also agree (up to multiplication by a constant) with the transformations which Chandrasekhar [2] and Sasaki and Nakamura [11] derived through a consideration of the theory of differential equations. When expanded in coordinates, (46) becomes

$$
\widehat{\psi}_{2 B}=\frac{2}{(l-1) l(l+1)(l+2)} \mathscr{L}_{1}^{\dagger} \mathscr{L}_{2}^{\dagger} \mathscr{D}_{0} \mathscr{D}_{0}\left(r^{2} \psi_{4 B}\right),
$$

while (48) becomes

$$
[(l-1) l(l+1)(l+2)+12 M i \sigma] \psi_{4 B}=\frac{\Delta^{2}}{2 r^{6}} \mathscr{L}_{-1} \mathscr{L}_{0} \mathscr{D}_{0}^{\dagger} \mathscr{D}_{0}^{\dagger}\left(r^{4} \widehat{\psi}_{2 B}\right) .
$$

It should be noted that the constant $[(l-1) l(l+1)(l+2)+12 M i \sigma]$ occurs naturally in our equation (60) or (48). (The radial part of the quantity $r^{3} \widehat{\psi}_{2 B}$ satisfies the RW ordinary differential equation.)

\section{Discussion}

The coordinate approach to the theory of transformation between the BP and RW equations provided by Chandrasekhar [2], and Sasaki and Nakamura [11], obscures how natural their results really are. As we have shown, the gauge invariant RW equation is a natural consequence of the Bianchi identities. Furthermore, the integrability conditions for the perturbed Bianchi identities also give rise to gauge invariant transformation identities relating RW and BP field variables. In fact there are two RW quantities which arise naturally in this way, one associated with $\psi_{4 B}$ and the other with $\psi_{0 B}$.

It is interesting to note the form of $\operatorname{Im}\left(\xi_{0}\right)$ in (52). As remarked previously, $\operatorname{Im}\left(\psi_{2 B}\right)$ is gauge invariant. If we add some quantity to $\psi_{2 B}$ so that the result is gauge invariant, we want to affect the real part of $\psi_{2 B}$ only, which on its own fails to be independent of gauge. As shown, $\operatorname{Im}\left(\xi_{0}\right)$ contains no new information and does not interfere with the gauge invariance of $\operatorname{Im}\left(\psi_{2 B}\right)$. So our ability to write $\operatorname{Im}\left(\xi_{0}\right)$ in the form of (52) agrees with our expectations.

The fact that $\operatorname{Im}\left(\xi_{0}\right)$ is nonzero in general means that the identities (54) and (55) relating $\psi_{0 B}$ and $\psi_{4 B}$ are not as simple as one would infer from Chandrasekhar [2]. After some work, and interpretation of $\overline{\psi_{4 B}}$ and $\overline{\psi_{0 B}}$, our identities agree with those 
of Chandrasekhar. Nevertheless, $\psi_{0 B}$ is uniquely determined, given $\psi_{4 B}$ (and vice versa), if we specify the time dependence.

The term $\xi_{0}-\xi_{0}^{\prime}$ which appears in our results, exits due to the lack of symmetry between the identities (34)-(37). This is something one does not encounter when treating the electromagnetic perturbations (see Teukolsky and Press [12], Fernandes and Lun [3]).

One may wonder if a similar approach provides gauge invariant quantities of spinweight \pm 1 . In fact, the answer is yes, and these will appear in Fernandes and Lun [3]. Actually these quantities allow the work presented here to be simplified greatly.

Importantly, the analysis presented here is completely valid in the flat space-time limit $M=0$ (that is, $\psi_{2}=0$ ). Thus, in particular, the wave equations ((24), (25), (38), (39)) and transformation identities ((34)-(37)) are the direct generalizations of their flat space-time forms to the Schwarzschild space-time. We are prompted to ask whether the approach outlined in this article can be further generalized to the Kerr background or indeed to other vacuum Petrov type D background metrics. Our preliminary results suggest that this is very likely, although the complexity of the NP commutators makes this work quite difficult.

\section{Appendices}

Below we present some of the missing detail from Section 2. For conciseness, when equations appear in pairs (related to each other by the prime operation - for example (22) and (23)) only the first will be considered here. The second can then be inferred.

\section{A1. Derivation of the transformation identities from the Bianchi identities.} Allowing $(\mathrm{p}-3 \varrho)$ to act on (6) and $\sigma^{\prime}$ to act on (5), using (2) and the NP commutators, and then eliminating $\psi_{3 B}$, we find

$$
(\mathrm{p}-3 \varrho)(\mathrm{p}-\varrho) \psi_{4 B}=\bar{\sigma}^{\prime} \tilde{\sigma}^{\prime} \psi_{2 B}+\left(\bar{\sigma}^{\prime} \tilde{\partial}_{B}^{\prime}+3 \bar{\partial}^{\prime} \pi_{B}-3 \mathrm{p} \lambda_{B}\right) \psi_{2} .
$$

Now from (13) this reduces to (26):

$$
(\mathrm{p}-3 \varrho)(\mathrm{p}-\varrho) \psi_{4 B}=\sigma^{\prime} \mathrm{J}^{\prime} \psi_{2 B}+\left(\bar{\sigma}^{\prime} \tilde{\partial}_{B}^{\prime}-3 \varrho \lambda_{B}-3 \mu \bar{\sigma}_{B}\right) \psi_{2} .
$$

The other identities (27)-(29) follow in a similar manner.

Now with the definition (30) for $\xi_{0}$, this equation may be expressed as (34):

$$
(\mathrm{p}-3 Q)(\mathrm{p}-Q) \psi_{4 B}=\mathbf{\partial}^{\prime} \boldsymbol{\partial}^{\prime}\left(\psi_{2 B}+\xi_{0}\right) \text {. }
$$

The verification of this is straightforward upon substitution, when we recall that

$$
\partial^{\prime} \sigma^{\prime} \text { } \boldsymbol{\prime} \equiv \frac{(l-1) l(l+1)(l+2)}{4 r^{4}}
$$


on a spin-weight -2 quantity, and $\bar{\sigma}^{\prime} r=0$ from (2).

After somewhat more work it can be shown that (27) and (35) are identical. To see this it is sufficient to show

$$
\left(\mathrm{p}^{\prime}+5 \mu\right)\left(\mathrm{p}^{\prime}+3 \mu\right)\left(\psi_{2 B}+\xi_{0}\right)=\delta \delta\left(\psi_{4 B}+\xi_{-2}\right) .
$$

The proof of this statement requires the perturbed and unperturbed NP commutation relations and Equations (2). Subsequently a term involving $\psi_{3 B}$ is introduced, and (6) is used to write this in terms of $\psi_{4 B}$. Derivatives of perturbed spin coefficients occur in precisely the correct combinations so that (15) and (19) allow rapid reduction, and the result follows.

A2. Reduction of (22) to the RW equation (38). The wave equation

$$
\begin{aligned}
& {\left[(\mathrm{p}-3 \varrho)\left(\mathrm{p}^{\prime}+3 \mu\right)-\delta \boldsymbol{\sigma}^{\prime}+3 \psi_{2}\right] \psi_{2 B}} \\
& \quad+\left[(\mathrm{p}-3 \varrho) \mathrm{p}_{B}^{\prime}-\delta \tilde{\sigma}_{B}^{\prime}-3 \mathrm{p}_{B} \mu+3 \varrho \mu_{B}+3 \mu \bar{\varrho}_{B}\right] \psi_{2}=0
\end{aligned}
$$

may be written in the form

$$
\left[(\mathrm{p}-3 \varrho)\left(\mathrm{p}^{\prime}+3 \mu\right)-\sigma \sigma^{\prime}+3 \psi_{2}\right]\left(\psi_{2 B}+\xi_{0}\right)=0
$$

by again using the perturbed and unperturbed NP commutators as well as (2), (19), (15), (6), (13), (16) and (17). The reduction of (23) to the RW equation (39) follows similarly.

A3. Gauge invariance. Since $\psi_{0 B}$ and $\psi_{4 B}$ are (both coordinate and tetrad) gauge invariant, the set of wave equations (24), (25), (38) and (39), together with the transformation identities (34)-(37) will be seen to be gauge invariant as well when we prove that the quantity

$$
\widehat{\psi}_{2 B}=\psi_{2 B}+\xi_{0}
$$

is gauge invariant. To prove that this is indeed true, consider a combined tetrad and coordinate gauge transformation. Then (Lun [5])

$$
\begin{gathered}
\psi_{2 B} \mapsto \psi_{2 B}-\left(X \mathrm{p}+Y \mathrm{p}^{\prime}\right) \psi_{2}, \\
\lambda_{B} \mapsto \lambda_{B}+\boldsymbol{\sigma}^{\prime} v, \\
\sigma_{B} \mapsto \sigma_{B}-\delta w
\end{gathered}
$$

and

$$
\tilde{\partial}_{B}^{\prime} \mapsto \tilde{\partial}_{B}^{\prime}+\left(\bar{\partial}^{\prime} X+v\right) \mathbf{p}+\left(\boldsymbol{\partial}^{\prime} Y+\bar{w}\right) \mathbf{p}^{\prime}
$$


when acting on $\psi_{2}$ (using (2)). A quick calculation reveals that all coordinate gauge terms $(X, Y)$ and tetrad gauge terms $(v, w)$ disappear. So $\widehat{\psi}_{2 B}$ is gauge independent, as is $\widehat{\psi}_{2 B}^{\prime}$.

This technique can be repeated for the quantities

$$
\widehat{\psi}_{2 B}-\widehat{\psi}_{2 B}^{\prime}=2 i \operatorname{Im}\left(\xi_{0}\right) \quad \text { and } \quad \widehat{\psi}_{2 B}-\left(\widehat{\psi}_{2 B}^{\prime}\right)=2 i \operatorname{Im}\left(\psi_{2 B}\right)
$$

\section{References}

[1] J. M. Bardeen and W. H. Press, "Radiation fields in the Schwarzschild background", J. Math. Phys. 14 (1973) 7-19.

[2] S. Chandrasekhar, The Mathematical Theory of Black Holes (Oxford University Press, New York, 1983).

[3] J. F. Q. Fernandes and A. W. C. Lun, J. Math. Phys. submitted.

[4] J. F. Q. Fernandes and A. W. C. Lun, in Proceedings of the Seventh Marcel Grossmann Meeting on General Relativity, (to appear).

[5] A. W. C. Lun, Ph. D. Thesis, Monash University, 1976.

[6] A. W.-C. Lun and E. D. Fackerell, "A master equation for perturbations to the Schwarzschild geometry", Lett. Cimento 9 (1974) 599-602.

[7] R. Penrose and W. Rindler, Spinors and Space-time. Volume I (Cambridge University Press, Cambridge, 1984).

[8] R. H. Price, "Nonspherical perturbations of relativistic gravitational collapse. I Scalar and gravitational perturbations", Phys. Rev. D 5 (1972) 2419-2438.

[9] R. H. Price, "Nonspherical perturbations of relativistic gravitational collapse. II Integer-spin, zero-rest-mass-fields", Phys. Rev. D 5 (1972) 2439-2454.

[10] T. Regge and J. A. Wheeler, "Stability of Schwarzschild singularity", Phys. Rev. 108 (1957) 1063.

[11] M. Sasaki and T. Nakamura, "Gravitational radiation from a Kerr black hole. I - Formulation and a method for numerical analysis", Prog. Theor. Phys. 67 (1982) 1788-1809.

[12] S. A. Teukolsky and W. H. Press, "Perturbations od a rotating black hole. III. interaction of the hole with gravitational and electromagnetic radiation", Astroph. J. 193 (1974) 443-461. 